\title{
Un cas de récupération ad integrum de la sensibilité-labio mentonnière suite à une complication per et post opératoire lors de l'avulsion d'une 3 e molaire mandibulaire.
}

\author{
Fauroux MA ${ }^{1}$, Desmyttere $\mathrm{M}^{2}$, Nieto $\mathrm{N}^{3}$, Torres $\mathrm{JH}^{4}$ \\ 1. Service d'Odontologie de Montpellier - Université Montpellier I : EA2415 \\ 2. Service d'Odontologie de Montpellier \\ 3. Service de Chirurgie-Maxillo-Faciale de Montpellier \\ 4. Service d'Odontologie de Montpellier - Université Montpellier I : EA4203
}

Un cas de récupération ad integrum de la sensibilité-labio mentonnière suite à une complication per et post opératoire lors de l'avulsion d'une 3e molaire mandibulaire Introduction : Les rapports anatomiques entre les $3 e$ molaires mandibulaires (3MM) et le paquet vasculo-nerveux alvéolaire inférieur sont parfois très étroits, et peuvent conduire à des complications nerveuses et hémorragiques au cours de l'avulsion de ces dents. Robert et al (1) ont estimé à $0,4 \%$ le taux moyen d'atteintes du nerf alvéolaire inférieur (NAI). La prescription de corticoïdes (AIS) a été évoquée pour diminuer ces complications et favoriser la neurorégénération (2). Ce travail présente le cas d'une récupération ad integrum de la sensibilité labio-mentonnière consécutive à l'atteinte du NAl suite à l'utilisation de la diathermocoagulation (DTC) pour juguler une hémorragie per-opératoire lors de l'avulsion d'une 3MM.

\section{Observation}

Une patiente âgée de 23 ans a été adressée pour avulsion des deux 3MM dans le cadre d'un suivi de prise en charge globale de fente labio-alvéolo-palatine opérée dans l'enfance. Aucun autre antécédent n'était à signaler. L'examen radiologique a objectivé une proximité des $3 \mathrm{MM}$ avec le NAI. Une information des complications et décisions thérapeutiques per-opératoires a été dispensée à la patiente. L'intervention s'est déroulée sous anesthésie générale du fait de l'anxiété de la patiente et d'un passé médical et chirurgical anxiogène. Lors du premier trait d'ostectomie vestibulaire, un saignement abondant immédiat a compromis la poursuite de l'intervention. Le site a fait l'objet d'une compression pendant 15 minutes, mais sans effet. La mise en place de cire à os a conduit à l'arrêt du saignement. A la reprise de l'intervention (séparation couronne/racine puis mobilisation et avulsion des fragments), le saignement a récidivé au fond de l'alvéole. La compression a été à nouveau inefficace. La DTC a entraîné l'arrêt définitif du saignement, mais au prix d'une atteinte du NAl, la patiente signalant une anesthésie labio-mentonnière droite complète à son réveil. Une prescription d'AIS a été immédiatement mise en place : $1 \mathrm{mg} / \mathrm{Kg} / \mathrm{j}$, en alternant $5 \mathrm{j}$ de prise et $7 \mathrm{j}$ ou $15 \mathrm{j}$ sans. Lors de contrôles à 1 semaine et 5 semaines, une cartographie de la zone anesthésiée a été réalisée et une diminution lente des symptômes a été notée. La prise d'AIS a été poursuivie jusqu'au contrôle à 10 semaines, où la restitution ad integrum de la sensibilité labio-mentonnière a été constatée.

\section{Discussion}

L'atteinte transitoire du NAl a probablement été liée à un échauffement par la DTC, ce qui a conduit à une neurapraxie post-opératoire. La restitution ad integrum de la sensibilité après une anesthésie totale est une évolution rare et a peut-être pu être potentialisée par l'utilisation prolongée des AIS.

Conclusion

L'avulsion des 3MM doit toujours faire l'objet d'une information quant aux complications opératoires. La neurapraxie est de bon pronostic contrairement à la section partielle ou complète du NAI.

mariealix.fauroux@free.fr

Références

(1) Robert et al. J Oral Maxillofac Surg, 2005,63:732-735

(2) Dan et al. J Oral Maxillofac Surg 2010,68:2207-2220

(C) The authors, published by EDP Sciences. This is an Open Access article distributed under the terms of the Creative Commons Attribution License 4.0 (http://creativecommons.org/licenses/by/4.0/) 